\title{
False Vacuum Decay in Rotating BTZ Spacetimes
}

\author{
Daiki Saito*and Chul-Moon Yoo ${ }^{\dagger}$ \\ Division of Particle and Astrophysical Science, Graduate School of Science, Nagoya \\ University, Nagoya 464-8602, Japan
}

\begin{abstract}
We analyse vacuum decay in rotating BTZ black hole spacetimes with the thin wall approximation. Possible parameter regions for the vacuum decay are clarified. We find that the nucleation rate is dominated by the bounce solution with the static shell configuration. The nucleation rate of the static shell decreases with the mass of the initial black hole. For a larger/smaller value of the initial black hole, the nucleation rate can be smaller/larger than that of the Coleman De Luccia vacuum decay in the pure AdS spacetime. Through the vacuum decay, the black hole gains its mass and loses the horizon area. We also find that the nucleation rate increases with increasing the angular momentum of the spacetime.
\end{abstract}

\section{Contents}

1 Introduction

2 Geometry of the Shell and Equations of Motion 3

2.1 Lorentzian and Euclidean Metric . . . . . . . . . . . . . . . . . . .

2.2 Bubble Wall as a Hypersurface . . . . . . . . . . . . . . . . . . . . . .

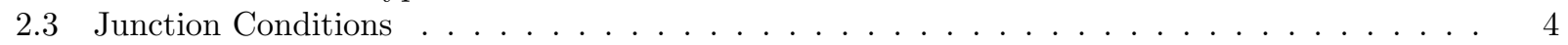

3 Conditions for the Existence of the Bounce Solution 6

3.1 Conditions for the Existence of Roots of $V(R)=0 \ldots \ldots \ldots \ldots \ldots$

3.2 Constraints from Time Parametrization . . . . . . . . . . . . . . . 7

3.3 Constraints from Horizon Condition ．.. . . . . . . . . . . . . . . . . 9

3.4 Allowed Region in the Parameter Space . . . . . . . . . . . . . . . . . . 9

4 Euclidean Action and the Decay Rate 11

5 Results 13

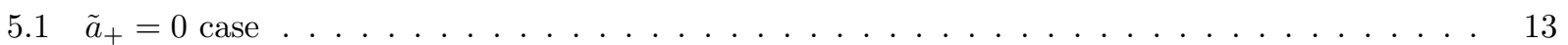

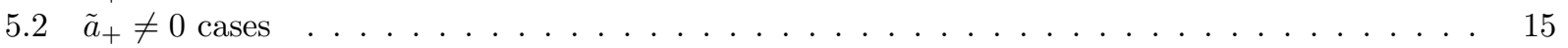

6 Summary and Discussion $\quad 17$

A Three-Dimensional Coleman De Luccia Instanton 17

\begin{tabular}{llr} 
B Static Shell & 19 \\
\hline
\end{tabular}

C Horizon Area $\quad 20$

*Email:saito.daiki.g3@s.mail.nagoya-u.ac.jp

${ }^{\dagger}$ Email:yoo@gravity.phys.nagoya-u.ac.jp 


\section{Introduction}

Vacuum decay is a transition from a classically stable state (false vacuum) to a lower energy state (true vacuum), which may be described by a quantum tunneling process. By considering this transition in the spacetime occupied by a false vacuum state, a true vacuum region surrounded by a bubble wall can be realized due to the quantum tunnelling. The nucleated bubble typically expands, and eventually, the spacetime will be filled with the true vacuum. This phenomenon was considered for the first time by Coleman over 40 years ago [1.2. Since gravity universally couples with other fields, it would be important to take into account the effect of gravity in vacuum decay. Pioneering work has been done by Coleman and De Luccia in Ref. 3, where the decay in a maximally symmetric spacetime was discussed. Vacuum decay in a black hole (BH) spacetime was firstly considered in Ref. 4], which stated that the four-dimensional Schwarzschild BH acts as a nucleation site for the decay, and more sophisticated researches have been performed in Refs. [5, 6. In particular, the authors in Refs. [5, 6] considered the cases in which the $\mathrm{BH}$ mass changes through the transition associated with the bubble nucleation, and reported that the mass and the horizon area of the $\mathrm{BH}$ may decrease in some cases.

As far as we know, the physical meaning of the vacuum decay in $\mathrm{BH}$ spacetimes has not yet been sufficiently clear. One plausible interpretation of the effect of a $\mathrm{BH}$ on the vacuum decay would be a thermal environmental system 7]. In Ref. 7], it is stated that the vacuum decay in the Schwarzschild (de-Sitter) spacetime can be regarded as the activated tunnelling in the flat background with the finite temperature equal to the Hawking temperature of the $\mathrm{BH}$. That is, the thermal radiation from the black hole supports the vacuum phase transition. On the basis of this interpretation, the effect of the black hole on the vacuum decay may involve the quantum nature of gravity. The decreasing horizon area reported in Ref. [5, 6] means the violation of the black hole area theorem in the classical theory and might also indicate the quantum gravity effect. Therefore, it might be expected that the vacuum decay in $\mathrm{BH}$ spacetimes is a clue to understanding the quantum nature of gravity.

Vacuum decay in BH spacetimes is also attractive from the aspect of several applications. The Higgs particle has been discovered in the LHC experiment in 2012 [8]9], and our universe might be in a false vacuum of Higgs potential $[10-16$. The estimated lifetime of the false vacuum in the flat background spacetime is longer than the age of the universe 17, 18. However, the lifetime can be decreased by the existence of BHs in the universe as is reported in Refs. $19 \mid 22$. Based on this result, by considering the effects of primordial black holes (PBHs) on the vacuum decay, Ref. 23] set constraints on the number and mass of PBHs.

From the aspect of the application, we should note that seed BHs are restricted to static and spherically symmetric ones in most researches. However, in general, BHs have non-zero values of the angular momentum, and the analyses with static and spherically symmetric solutions would not be sufficient for the application in our universe. In order to get a deeper insight into the fundamental understanding and general property of the vacuum decay in $\mathrm{BH}$ spacetimes, it would be helpful to collect more variety of vacuum decay phenomena in $\mathrm{BH}$ spacetimes. In particular, in this paper, we focus on the effects of the angular momentum. In four-dimensional spacetimes, however, the analyses of vacuum decay without spherical symmetry become significantly difficult because the bubble dynamics cannot be reduced to one-dimensional particle mechanics as in Ref. [5]. No formal procedure is known for treating the bubble nucleation in a non-spherical BH spacetime, and there can be found only one attempt in Ref. 24] with some assumptions. In this paper, in order to see effects of the angular momentum without the difficulty, we consider the vacuum decay in rotating BTZ BH spacetimes, which are three-dimensional asymptotically AdS spacetimes. In the three-dimensional asymptotically AdS spacetime, the BH spin does not violate the spherical symmetry, and we can proceed in the same way as Ref. [5.

This paper is organized as follows. In Sec. 2, we see the Euclidean metric of the BTZ spacetime and consider the matching conditions for two BTZ spacetimes separated by a thin spherical shell. The shell equations of motion are derived in Sec. 2.3 and we show that the shell dynamics is reduced to a onedimensional potential problem of particle dynamics. In Sec. 3. we clarify the possible parameter regions for 
the bubble nucleation. In Sec. 4, we derive the explicit expression of the vacuum decay rate and show the results in Sec. 5. Sec. 6 is devoted to a summary and conclusion.

Throughout this paper, we use the geometrized units in which both the speed of light and the Newton's gravitational constant are unity, $G=c=1$. 1

\section{Geometry of the Shell and Equations of Motion}

\subsection{Lorentzian and Euclidean Metric}

The line element in the BTZ spacetime is given as follows in the Boyer-Lindquist coordinates 25,26 :

$$
d s^{2}=-f(r) d t^{2}+\frac{1}{f(r)} d r^{2}+r^{2}\left(d \varphi-\frac{4 J}{r^{2}} d t\right)^{2}
$$

with

$$
f(r)=-8 M+\frac{r^{2}}{l^{2}}+\frac{16 J^{2}}{r^{2}} .
$$

Here, $M$ and $J$ are the mass and the angular momentum of the BH, respectively, and $l$ is the AdS length, which is given by $l^{-2}=-\Lambda$ with $\Lambda$ being the cosmological constant.

In order to evaluate the decay rate, we need to calculate geometric quantities in the Euclidean spacetime. We can get the Euclidean metric through the transformations $t=-i t_{E}$ and $J=-i J_{E}$ :

$$
d s^{2}=f_{E}(r) d t_{E}^{2}+\frac{1}{f_{E}(r)} d r^{2}+r^{2}\left(d \varphi+\frac{4 J_{E}}{r^{2}} d t_{E}\right)^{2}
$$

where

$$
f_{E}(r)=-8 M+\frac{r^{2}}{l^{2}}-\frac{16 J_{E}^{2}}{r^{2}}
$$

This paper focuses on vacuum decay in the BTZ spacetime with the thin wall approximation. We assume that the spacetime is spherically symmetric, and therefore the spacetime after the bubble nucleation is also described by a BTZ spacetime. Since the shape of the bubble is also spherical, we can express the bubble wall trajectory as $r=R(t)$. Then, for convenience, we define a frame that is co-rotating with the spacetime on the bubble wall, that is, the non-diagonal component of the metric vanishes on the bubble wall. Following Ref. 27, we transform the azimuthal coordinate $\varphi$ to $\phi$ defined by

$$
\begin{aligned}
d \phi & :=\left.\left(d \varphi+\frac{g_{t \varphi}}{g_{\varphi \varphi}} d t\right)\right|_{r=R(t)} \\
& =d \varphi-\frac{4 J}{R^{2}(t)} d t
\end{aligned}
$$

and rewrite the metric as follows:

$$
\begin{aligned}
d s^{2} & =\left\{-f(r)+16 r^{2} J^{2}\left(\frac{1}{R^{2}(t)}-\frac{1}{r^{2}}\right)^{2}\right\} d t^{2}+\frac{1}{f(r)} d r^{2}+r^{2} d \phi^{2}+8 J r^{2}\left(\frac{1}{R^{2}(t)}-\frac{1}{r^{2}}\right) d t d \phi \\
& =\left\{f_{E}(r)+16 r^{2} J_{E}^{2}\left(\frac{1}{R^{2}\left(t_{E}\right)}-\frac{1}{r^{2}}\right)^{2}\right\} d t_{E}^{2}+\frac{1}{f_{E}(r)} d r^{2}+r^{2} d \phi^{2}-8 J_{E} r^{2}\left(\frac{1}{R^{2}(t)}-\frac{1}{r^{2}}\right) d t_{E} d \phi
\end{aligned}
$$

\footnotetext{
${ }^{1}$ Note that many papers treat BTZ spacetime with BTZ unit, $8 G=c=1$.
} 


\subsection{Bubble Wall as a Hypersurface}

The bubble wall is given as a hypersurface $\mathcal{W}: r-R\left(t_{E}\right)=0$ whose unit normal vector $n_{\mu}$ is given by

$$
n_{\mu}=C_{E}\left(-\partial_{t_{E}} R, 1,0\right)
$$

with

$$
C_{E}=\left[\frac{1}{f_{E}(R)}\left(\partial_{t_{E}} R\right)^{2}+f_{E}(R)\right]^{-1 / 2}
$$

in the coordinate bases associated with $\left(t_{E}, r, \phi\right)$. We use $\phi$ and the proper time $\tau$ of the radial observer on the bubble wall as the intrinsic coordinates of the wall. The four-velocity of the radial observer can be written as

$$
v^{\mu}=\left(\dot{t}_{E}, \dot{R}, 0\right),
$$

where the dot denotes the derivative with respect to $\tau$. From the normalization condition of the four velocity $\left.g_{E \mu \nu} v^{\mu} v^{\nu}\right|_{r=R(t)}=1$, we obtain

$$
f_{E}(R) \dot{t}_{E}=\sqrt{f_{E}(R)-\dot{R}^{2}} .
$$

From this Eq. (2.11), we obtain $C_{E}=\dot{t}_{E}$, and can rewrite the normal vector as

$$
n_{\mu}=\left(-\dot{R}, \dot{t}_{E}, 0\right) \text {. }
$$

The components of the projection tensor onto $\mathcal{W}$ are given by

$$
\begin{aligned}
& e_{\tau}^{\mu}=v^{\mu}=\left(\dot{t}_{E}, \dot{R}, 0\right), \\
& e_{\phi}^{\mu}=(0,0,1) .
\end{aligned}
$$

\subsection{Junction Conditions}

In this subsection, we derive equations of the shell motion from the Israel's junction condition [28. We label physical quantities on the spacetime before/after the nucleation with the subscript $+/-$. The junction condition consists of the 1st junction condition

$$
\left[h_{a b}\right]_{ \pm}=0
$$

and the 2nd junction condition

$$
\left[K_{a b}\right]_{ \pm}=-8 \pi\left(S_{a b}-h_{a b} S\right),
$$

where, for convenience, we used the expression

$$
[A]_{ \pm}:=A_{+}-A_{-},
$$

and $S_{a b}$ is the energy-momentum tensor on the shell. $h_{a b}$ and $K_{a b}$ are the induced metric and the extrinsic curvature on the shell, respectively. In the Euclidean spacetime, they are defined as

$$
\begin{aligned}
h_{E a b} & :=e_{a}^{\mu} e_{b}^{\nu} g_{E \mu \nu}, \\
K_{E a b} & :=e_{a}^{\mu} e_{b}^{\nu} \nabla_{\mu} n_{\nu} .
\end{aligned}
$$


In this paper, we assume that the energy-momentum on the shell is given as follows:

$$
S_{E a b}=-\sigma h_{E a b}
$$

where $\sigma$ is the value of the tension. In the current situation, the induced metric is

$$
h_{E \pm a b}=\left(\begin{array}{cc}
1 & 0 \\
0 & R^{2}
\end{array}\right)
$$

and Eq. (2.15) is automatically satisfied. From Eq. (2.20), we can rewrite Eq. (2.16) as

$$
\left[K_{E a b}\right]_{ \pm}=-8 \pi \sigma h_{E a b} .
$$

Let us calculate the components of the symmetric tensor $K_{E a b}$. From the $(\tau, \phi)$ component of Eq. 2.19), we get

$$
K_{E \tau \phi}=-4 f_{E} J_{E} \frac{r}{R^{2}} \dot{t}_{E}^{2}-\frac{4 J_{E}}{f_{E}} \frac{1}{r} \dot{R}^{2},
$$

where we used the following expressions of each component of the Christoffel symbol

$$
\begin{aligned}
\Gamma_{t_{E} \phi}^{r} & =4 f_{E} J_{E} \frac{r}{R^{2}}, \\
\Gamma_{r \phi}^{t_{E}} & =-\frac{4 J_{E}}{f_{E}} \frac{1}{r} .
\end{aligned}
$$

Evaluating Eq. 2.23) on the shell with Eq. 2.11), we get

$$
\left.K_{E \tau \phi}\right|_{\mathcal{W}}=-\frac{4 J_{E}}{R}
$$

Because $h_{E \tau \phi}=0$, the $(\tau, \phi)$ component of Eq. 2.22 is equivalent to

$$
\begin{gathered}
-\frac{4 J_{E+}}{R}+\frac{4 J_{E-}}{R}=0, \\
\therefore J_{+}=J_{-} .
\end{gathered}
$$

Obviously, this is the consequence of the conservation of the angular momentum. The $(\phi, \phi)$ component of the extrinsic curvature is

$$
K_{E \phi \phi}=f_{E} r \dot{t}_{E}
$$

where we used

$$
\Gamma_{\phi \phi}^{r}=-f_{E} r .
$$

By using Eq. 2.11), the $(\phi, \phi)$ component of Eq. 2.22 can be written as

$$
\sqrt{f_{E+}-\dot{R}^{2}}-\sqrt{f_{E-}-\dot{R}^{2}}=-8 \pi \sigma R .
$$

Solving this for $\dot{R}^{2}$, we get

$$
-\frac{1}{2} \dot{R}^{2}=V(R):=\frac{1}{2} \bar{\sigma}^{2} R^{2}-\frac{1}{2} \bar{f}_{E}+\frac{\left(\Delta f_{E}\right)^{2}}{32 \bar{\sigma}^{2} R^{2}},
$$

where, we defined $\bar{\sigma}:=4 \pi \sigma$ and

$$
\bar{A}:=\frac{A_{+}+A_{-}}{2} .
$$


Therefore the shell dynamics reduces to the one-dimensional potential problem. The potential form can be rewritten as follows:

$$
2\left(\frac{R}{l_{-}}\right)^{2} V(R)=A\left(\frac{R}{l_{-}}\right)^{4}+B\left(\frac{R}{l_{-}}\right)^{2}+C
$$

where

$$
\begin{aligned}
A & :=s^{2}-\frac{1}{2}\left(\frac{1}{L_{+}^{2}}+1\right)+\frac{1}{16 s^{2}}\left(\frac{1}{L_{+}^{2}}-1\right)^{2}, \\
B & :=8 \bar{M}-\frac{\Delta M}{s^{2}}\left(\frac{1}{L_{+}^{2}}-1\right) \\
C & :=-16\left(M_{+} L_{+} \tilde{a}_{+}\right)^{2}+\frac{4(\Delta M)^{2}}{s^{2}} .
\end{aligned}
$$

Here, we wrote $V(R)$ as a function of $R / l_{-}$with the five dimensionless parameters: $M_{+}, M_{-}, \tilde{a}_{+}:=$ $J /\left(M_{+} l_{+}\right), L_{+}:=l_{+} / l_{-}$and $s:=\bar{\sigma} l_{-}$. The functional form of $V(R)$ is depicted in Fig. 1 for $M_{+}=0.1$, $M_{-}=0.33, \tilde{a}_{+}=0, L_{+}=2.0$ and $s=0.2$. The Euclidean shell can oscillate between the two roots of $V(R)=0$, at which the velocity $\dot{R}$ of the shell vanishes. The bubble emerges with the radius determined by the condition $\dot{R}=0$ or equivalently $V(R)=0$. When we have two roots of $V(R)=0$, there are two possible radii of the bubble at the moment of the nucleation. They share the bounce solution and have the same value of the nucleation rate. After the nucleation, the shell motion obeys the junction condition in the Lorentz spacetime, and is described by the one-dimensional motion under the potential $-V(R)$. The shell is allowed to move in the region $-V(R)<0$. The shell which emerges at the larger root of $V(R)=0$ expands, and the true vacuum region also expands. We call it an expanding solution. On the other hand, the shell which emerges at the smaller root shrinks, and the false vacuum region covers the whole region again. We call it a shrinking solution. It is worthy to note that, in the shrinking solution, through the shell accretion, the horizon area must increase due to the horizon area law, and the mass of the $\mathrm{BH}$ must be identical to $M_{+}$due to the energy conservation. This fact indicates that the horizon area must decrease through the bubble nucleation. We will see this is actually realized in the current situation, and the decreasing horizon area indicates the quantum nature of the bubble nucleation (see App. C for details.).

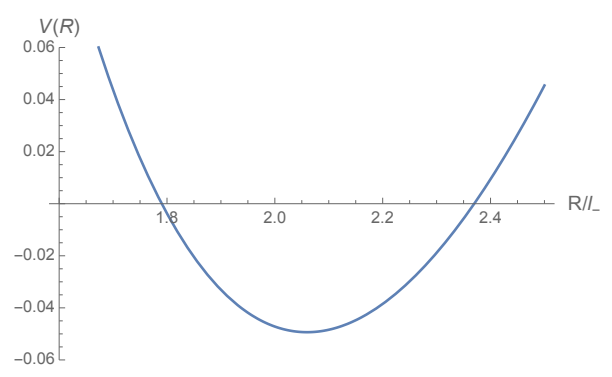

Figure 1: The potential form for $M_{+}=0.1, M_{-}=0.33, \tilde{a}_{+}=0, L_{+}=2.0$ and $s=0.2$

\section{Conditions for the Existence of the Bounce Solution}

In order for the bubble nucleation process to exist and describe a physically reasonable process, several conditions must be satisfied. We list all the conditions and summarize the allowed region of the parameters in this section (see Refs. 29, 30 for the coexistence of two BTZ spacetimes connected by thin-branes). 


\subsection{Conditions for the Existence of Roots of $V(R)=0$}

For the shell to oscillate, we need a finite $V(R)<0$ region in $R>0$. From Eq. 2.33), we can regard $R^{2} V(R)$ as a quadratic function for $R^{2}$. For the functional form of $V(R)$ to be convex downward, the condition $A>0$ is required. In addition, we need two real positive roots of $R^{2} V(R)=0$ for $R^{2}$ (see App. D for other possibilities). This condition requires $C>0,-B / 2 A>0$ and $B^{2}-4 A C>0$. To summarize, we need $A \cdots D$ satisfying the following four conditions:

$$
\begin{aligned}
A & >0, \\
B & <0, \\
C & >0, \\
D:=B^{2}-4 A C & \geq 0 .
\end{aligned}
$$

From Eq. 3.1], we get

$$
s^{2}-\frac{1}{2}\left(\frac{1}{L_{+}^{2}}+1\right)+\frac{1}{16 s^{2}}\left(\frac{1}{L_{+}^{2}}-1\right)^{2}>0
$$

or equivalently,

$$
s^{2}<\frac{1}{4 L_{+}^{2}}-\frac{1}{2 L_{+}}+\frac{1}{4}, \frac{1}{4 L_{+}^{2}}+\frac{1}{2 L_{+}}+\frac{1}{4}<s^{2} .
$$

Eq. (3.2 requires $(\Delta \Lambda)(\Delta M)<0$. In vacuum decay, we assume $\Lambda_{+}>\Lambda_{-}$, and obtain $M_{+}<M_{-}$as a necessary condition. In other words, the mass must decrease to form a bounce solution. Moreover, Eq. (3.2) imposes the lower bound for $M_{-}$:

$$
M_{-}>M_{B}:=M_{+} \frac{1-L_{+}^{2}\left(1+4 s^{2}\right)}{1-L_{+}^{2}\left(1-4 s^{2}\right)}
$$

Eq. 3.3 can be written as follows:

$$
-16\left(M_{+} L_{+} \tilde{a}_{+}\right)^{2}+\frac{4(\Delta M)^{2}}{s^{2}}>0 .
$$

This gives the condition for $M_{-}$under fixed values of $L_{+}, s, \tilde{a}_{+}$and $M_{+}$:

$$
M_{-}>M_{C}:=M_{+}\left(1+2 s L_{+} \tilde{a}_{+}\right) .
$$

Another condition for the mass also stems from Eq. (3.4):

$$
\begin{aligned}
& M_{-} \geq M_{D}:=\frac{M_{+}}{2}\left[1+L_{+}^{2}\left(1-4 s^{2}\right)+\sqrt{\left(1-\tilde{a}_{+}^{2}\right)\left\{1+L_{+}^{4}\left(1-4 s^{2}\right)^{2}-2 L_{+}^{2}\left(1+4 s^{2}\right)\right\}}\right] \\
& M_{-} \leq M_{D}^{\prime}:=\frac{M_{+}}{2}\left[1+L_{+}^{2}\left(1-4 s^{2}\right)-\sqrt{\left(1-\tilde{a}_{+}^{2}\right)\left\{1+L_{+}^{4}\left(1-4 s^{2}\right)^{2}-2 L_{+}^{2}\left(1+4 s^{2}\right)\right\}}\right] .
\end{aligned}
$$

When $D=0$, the shell can exist only at the radius of the degenerate root of $V(R)=0$, that is, the shell has a constant radius with time. We call it a static shell.

\subsection{Constraints from Time Parametrization}

In addition to the conditions for the potential form, there are other conditions for the motion of the shell to be physical. Specifically, the shell motion must be "future-directed" in both sides of the shell, that is, $\dot{t}_{E \pm} \geq 0$. We can rewrite these requirements as

$$
\begin{aligned}
& f_{E+}(R) \dot{t}_{E+}=\sqrt{f_{E+}(R)-\dot{R}^{2}}=-\bar{\sigma} R-\frac{\Delta f_{E}}{4 \bar{\sigma} R} \geq 0, \\
& f_{E-}(R) \dot{t}_{E-}=\sqrt{f_{E-}(R)-\dot{R}^{2}}=\bar{\sigma} R-\frac{\Delta f_{E}}{4 \bar{\sigma} R} \geq 0
\end{aligned}
$$


where we used Eq. (2.11).

The inequality (3.11) is equivalent to

$$
\left(\frac{R}{l_{-}}\right)\left[-s-\frac{L_{+}^{-2}-1}{4 s}\right]+\frac{1}{R / l_{-}} \frac{2 \Delta M}{s} \geq 0 .
$$

From the discussion in the previous subsection, we necessarily have $\Delta M<0$. Hence, for the inequality (3.13) to be satisfied, the coefficient of $R$ must be positive. From this, we obtain the constraint for $s$ :

$$
s^{2}<\frac{1}{4}-\frac{1}{4 L_{+}^{2}}
$$

The inequality 3.13$)$ is saturated when

$$
\frac{R}{l_{-}}=\frac{R_{+}^{*}}{l_{-}}:=\sqrt{\frac{2 \Delta M}{s\left(s+\frac{L_{+}^{-2}-1}{4 s}\right)}},
$$

and the shell must oscillate between two radii larger than $R_{+}^{*}$. This requires $\left(R_{+}^{*}\right)^{2} V\left(R_{+}^{*}\right)>0$ and $\left.\frac{d}{d R}\left[R^{2} V(R)\right]\right|_{R=R_{+}^{*}}<0$. These conditions are equivalent to

$$
\begin{aligned}
& \frac{\tilde{a}_{+}^{2} M_{+}^{2}\left(-1+L_{+}^{2}\left(1-4 s^{2}\right)\right)^{2}+4\left(M_{-}-M_{+}\right)\left(M_{-}+L_{+}^{2} M_{+}\left(-1+4 s^{2}\right)\right)}{\left(M_{-}-M_{+}\right)\left(1+L_{+}^{2}\left(-1+4 s^{2}\right)\right)}>0, \\
& 4 M_{+}+\frac{8\left(M_{-}-M_{+}\right)}{1+L_{+}^{2}\left(-1+4 s^{2}\right)}<0 .
\end{aligned}
$$

We may consider the condition 3.12 in a similar way to that for the condition (3.11). First, we rewrite the inequality (3.12) as

$$
\left(\frac{R}{l_{-}}\right)\left[s-\frac{L_{+}^{-2}-1}{4 s}\right]+\frac{1}{R / l_{-}} \frac{2 \Delta M}{s} \geq 0 .
$$

We find that the coefficient of $R$ is positive for any $s>0$. The inequality $(3.18)$ is saturated when

$$
\frac{R}{l_{-}}=\frac{R_{-}^{*}}{l_{-}}:=\sqrt{\frac{-2 \Delta M}{s\left(s-\frac{L_{+}^{-2}-1}{4 s}\right)}},
$$

and the region of the motion must be larger than $R_{-}^{*}$. Hence we require $\left(R_{-}^{*}\right)^{2} V\left(R_{-}^{*}\right)>0$ and $\left.\frac{d}{d R}\left[R^{2} V(R)\right]\right|_{R=R_{-}^{*}}<$ 0 . However, we see that $\frac{R_{+}^{*}}{l_{-}}>\frac{R_{-}^{*}}{l_{-}}$from

$$
\left(\frac{R_{+}^{*}}{l_{-}}\right)^{2}-\left(\frac{R_{-}^{*}}{l_{-}}\right)^{2}=\frac{4 \Delta M}{\left(s+\frac{L_{+}^{-2}-1}{4 s}\right)\left(s-\frac{L_{+}^{-2}-1}{4 s}\right)}>0 .
$$

Therefore it is sufficient to require only $\left(R_{+}^{*}\right)^{2} V\left(R_{+}^{*}\right)>0$ and $\left.\frac{d}{d R}\left[R^{2} V(R)\right]\right|_{R=R_{+}^{*}}<0$. By solving Eqs. 3.16 and (3.17), we obtain

$$
\begin{aligned}
& M_{-}<M_{T}:=\frac{M_{+}}{2}\left[1+L_{+}^{2}\left(1-4 s^{2}\right)+\sqrt{\left(1-\tilde{a}_{+}^{2}\right)\left\{1+L_{+}^{2}\left(-1+4 s^{2}\right)\right\}^{2}}\right] \\
& M_{-}>M_{T}^{\prime}:=\frac{M_{+}}{2}\left[1+L_{+}^{2}\left(1-4 s^{2}\right)\right] .
\end{aligned}
$$




\subsection{Constraints from Horizon Condition}

In addition to the above conditions, we impose the condition that the shell oscillates outside the event horizon. The outer horizon radius is given by the roots of $f_{E}(r)=0$, which can be written as ${ }^{2}$

$$
r_{\mathcal{H}_{ \pm}}=\sqrt{4 M_{ \pm} l_{ \pm}^{2}+4 l_{ \pm} \sqrt{M_{ \pm}^{2} l_{ \pm}^{2}-J^{2}}}
$$

Since $r_{\mathcal{H}}<r_{\mathcal{H}_{+}}$from other conditions (see App. C), it is sufficient to require that the shell oscillates between two radii larger than $r_{\mathcal{H}_{+}}$. That is, we impose the following conditions: $V\left(r_{\mathcal{H}_{+}}\right) \geq 0$ and $\left(r_{\mathcal{H}_{+}}\right)^{2}<-B / 2 A$. While the former puts no constraint on the parameters, from the latter, we obtain the inequality for $M_{-}$:

$$
M_{-}>M_{R}:=-M_{+} \frac{-1+\sqrt{1-\tilde{a}_{+}^{2}}+\left(1+\sqrt{1-\tilde{a}_{+}^{2}}\right) L_{+}^{4}\left(1-4 s^{2}\right)^{2}-2 \sqrt{1-\tilde{a}_{+}^{2}} L_{+}^{2}\left(1+4 s^{2}\right)}{2+L_{+}^{2}\left(-2+8 s^{2}\right)} .
$$

\subsection{Allowed Region in the Parameter Space}

In this subsection, we summarize the above conditions and show some examples of the allowed region for the parameters. First, we consider the conditions (3.6) and (3.14) for $L_{+}$and $s$, and fix them. Next, we fix $\tilde{a}_{+}$, and show the allowed region for $M_{+}$and $M_{-}$by considering Eqs. (3.7), (3.9), 3.10) and 3.21).

On the two conditions of $\sqrt{3.6}$, only the second one, $s^{2}<\frac{1}{4 L_{+}^{2}}-\frac{1}{2 L_{+}}+\frac{1}{4}$, can be compatible with the condition 3.14. With $L_{+}>1$, we see that

$$
\left(-\frac{1}{4 L_{+}^{2}}+\frac{1}{4}\right)-\left(\frac{1}{4 L_{+}^{2}}-\frac{1}{2 L_{+}}+\frac{1}{4}\right)=\frac{1}{2}\left(\frac{1}{L_{+}}-\frac{1}{L_{+}^{2}}\right)>0 .
$$

By using this Eq. 3.25) and $s>0$, we eventually find

$$
s<\frac{1}{2}-\frac{1}{2 L_{+}}
$$

for a given value of $L_{+}$. From this condition, we can simplify the expression $M_{T}$ as

$$
M_{T}=\frac{M_{+}}{2}\left[1+L_{+}^{2}\left(1-4 s^{2}\right)-\sqrt{1-\tilde{a}_{+}^{2}}\left\{1+L_{+}^{2}\left(-1+4 s^{2}\right)\right\}\right] .
$$

Setting $L_{+}=2.0, s=0.2$ and $\tilde{a}_{+}=0.0$, we obtain the allowed regions indicated by the conditions (3.7), (3.9), 3.10 and (3.21) as the shaded regions in Figs. 2 and Fig 3 . From Figs. 2 and 3 , we see that the allowed region cannot exist in the region $M_{-}<M_{D}^{\prime}$ and we have to take the branch $M_{-}>M_{D}$.

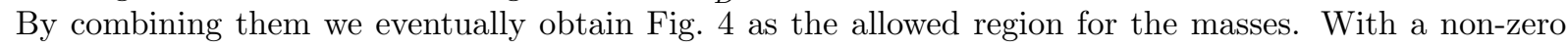
angular momentum, for example $\tilde{a}_{+}=0.2,0.5$, we get Fig. 5 . Regardless of the value of the angular momentum, the lower bound of $M_{-}$is given by that of the static shell.

\footnotetext{
${ }^{2}$ Subscript $+/$ - indicates the horizon radius before/after the nucleation, not the outer/inner horizon. From this expression, we can see that the condition for the extremal $\mathrm{BH}$ is $J / l=M$.
} 


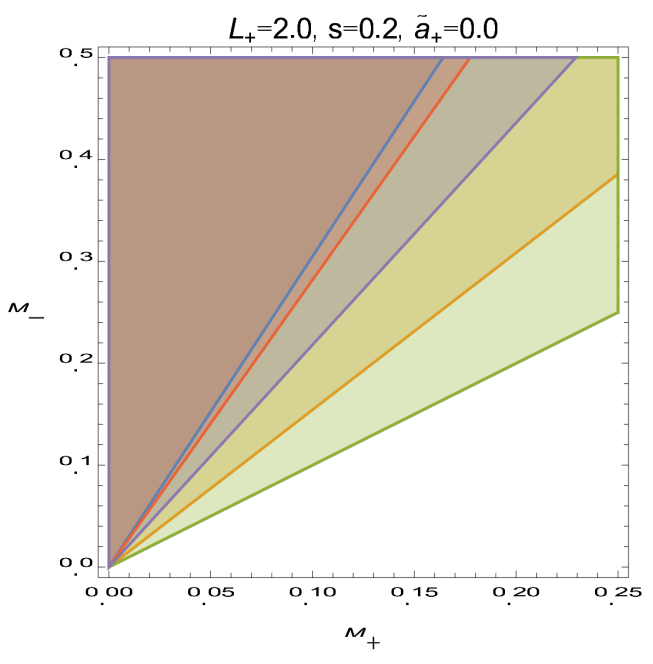

Figure 2: The lower bound of $M_{-}$

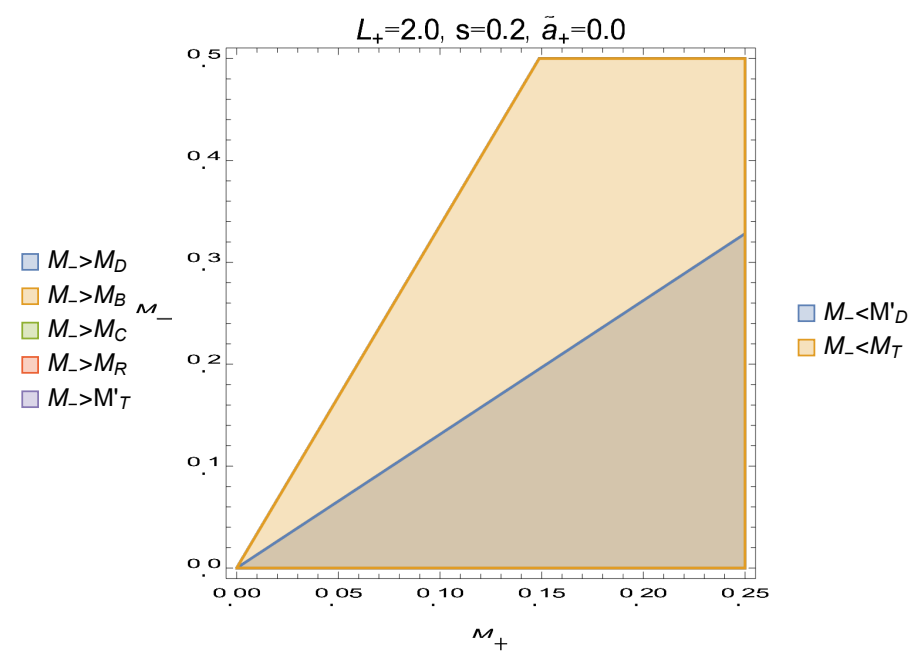

Figure 3: The upper bound of $M_{-}$

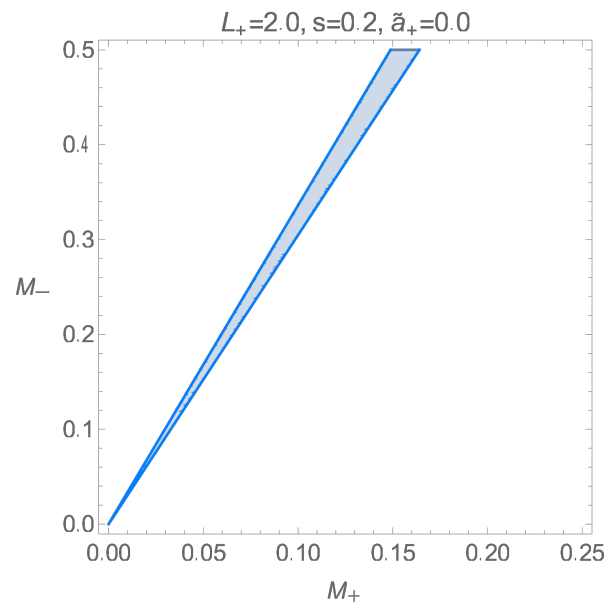

Figure 4: The allowed region $\left(\tilde{a}_{+}=0.0\right)$ 

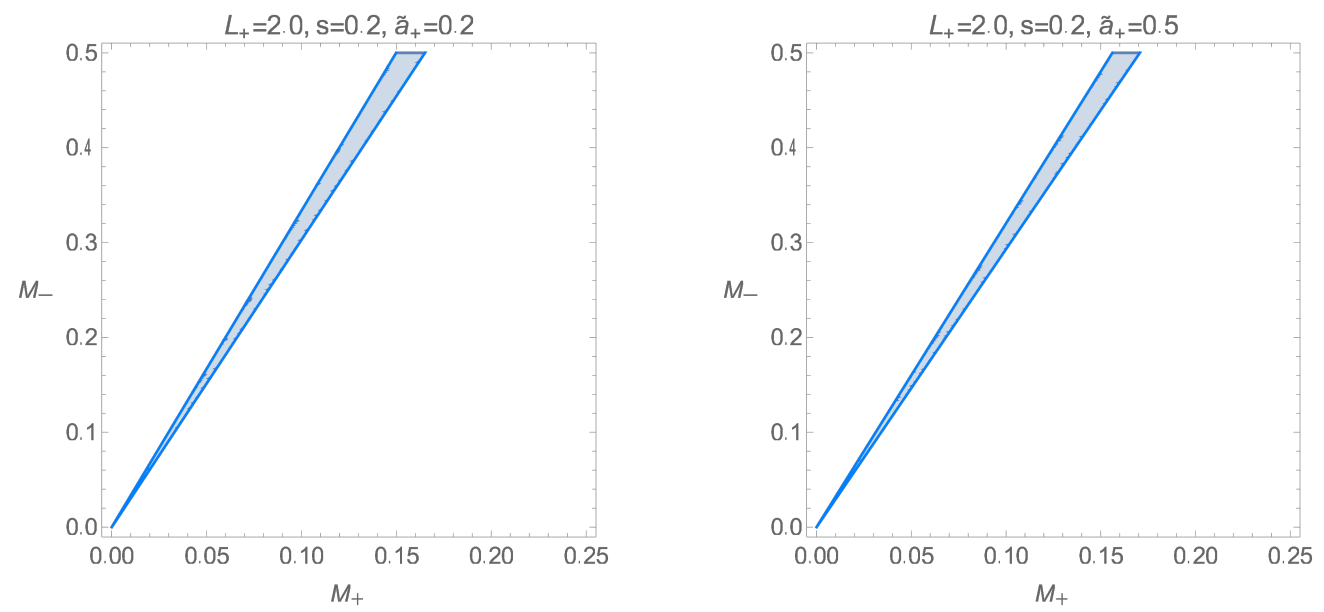

Figure 5: The allowed region $\left(\tilde{a}_{+} \neq 0.0\right)$

\section{Euclidean Action and the Decay Rate}

According to Ref. [1], the decay rate per unit of time and volume $\Gamma$ is given by

$$
\Gamma \propto e^{-\mathcal{B} / \hbar},
$$

where the value of $\mathcal{B}$ is given by the difference between the values of the Euclidean action of the bounce solution $S_{E}$ and the false vacuum $S_{E 0}$ as $\mathcal{B}:=S_{E}-S_{E 0}$. Since the main contribution to the decay rate comes from the exponential part, ignoring the factor of the dependence, we focus on the value of $\mathcal{B}$. Then we can estimate the decay rate by calculating the Euclidean actions. In this section, we derive an analytic form of $\mathcal{B}$ in the BTZ case.

$S_{E}$ can be divided into the contributions from the horizon $S_{\mathcal{H}}$, bubble wall $S_{\mathcal{W}}$ and bulk $S_{\mathcal{M}_{ \pm}}$as follows:

$$
S_{E}=S_{\mathcal{H}}+S_{\mathcal{M}_{+}}+S_{\mathcal{M}_{-}}+S_{\mathcal{W}}
$$

$S_{\mathcal{H}}$ can be evaluated by considering the action in the vicinity of the horizon (see Appendices in Refs. [5 24]). In the current setting, the value of $S_{\mathcal{H}}$ is given as

$$
S_{\mathcal{H}}=-\frac{A_{\mathcal{H}_{-}}}{4},
$$

where $A_{\mathcal{H}_{-}}$is the area of the horizon. $S_{\mathcal{W}}$ can be written as

$$
\begin{aligned}
S_{\mathcal{W}} & =-\int_{\mathcal{W}} d^{2} x \sqrt{h_{E}} \int_{R-0}^{R+0} d r \mathcal{L}_{m} \\
& =\int_{\mathcal{W}} d^{2} x \sqrt{h_{E}} \int_{R-0}^{R+0} d r \sigma \delta\left(r-R\left(t_{E}\right)\right) \\
& =\int_{\mathcal{W}} d^{2} x \sqrt{h_{E}} \sigma
\end{aligned}
$$

$S_{\mathcal{M}_{ \pm}}$can be written as

$$
S_{\mathcal{M}_{ \pm}}=-\int_{\mathcal{M}_{ \pm}} d^{3} x \sqrt{g_{E \pm}}\left(\frac{1}{16 \pi}^{3} \mathcal{R}^{(E)}+\mathcal{L}_{m}^{(E)}\right)+\frac{1}{8 \pi} \int_{\partial \mathcal{M}_{ \pm}} d^{2} x \sqrt{h_{E}} \tilde{K}_{E \pm}
$$


where the second term is the boundary term. With the ADM decomposition, we obtain

$$
\begin{aligned}
S_{\mathcal{M}_{ \pm}} & =-\frac{1}{16 \pi} \oint d t_{E \pm} \int_{\Sigma_{t_{E \pm}}} d^{2} x \sqrt{g_{E \pm}}\left({ }^{2} \mathcal{R}^{(E)}-\tilde{K}_{E \pm}^{2}+\tilde{K}_{E \pm a b} \tilde{K}_{E \pm}^{a b}+16 \pi \mathcal{L}_{m}^{(E)}\right) \\
& +\frac{1}{8 \pi} \int_{\mathcal{W}} d^{2} x \sqrt{h_{E}} \tilde{K}_{E \pm}+\frac{1}{8 \pi} \int_{\mathcal{W}} d^{2} x \sqrt{h_{E}} \tilde{n}_{ \pm \mu} \tilde{u}_{ \pm}^{\nu} \nabla_{\nu} \tilde{u}_{ \pm}^{\mu},
\end{aligned}
$$

where $\tilde{u}_{ \pm}^{\mu}$ is the unit normal vector of the constant time slice $\Sigma_{t_{E \pm}}, \tilde{n}_{ \pm \mu}$ is the ingoing unit normal vector of $\mathcal{W}$, and $\tilde{K}_{E \pm}= \pm K_{ \pm}$is the Euclidean extrinsic curvature associated with $\tilde{n}_{ \pm \mu}$. The first line vanishes from the Hamiltonian constraint ${ }^{2} \mathcal{R}^{(E)}-\tilde{K}_{E \pm}^{2}+\tilde{K}_{E \pm a b} \tilde{K}_{E \pm}^{a b}+16 \pi \mathcal{L}_{m}^{(E)}=0$, and we obtain

$$
S_{\mathcal{M}_{+}}+S_{\mathcal{M}_{-}}=\frac{1}{8 \pi} \int_{\mathcal{W}} d^{2} x \sqrt{h_{E}}\left(K_{+}-K_{-}\right)+\frac{1}{8 \pi} \int_{\mathcal{W}} d^{2} x \sqrt{h_{E}}\left(n_{+\mu} \tilde{u}_{+}^{\nu} \nabla_{\nu} \tilde{u}_{+}^{\mu}-n_{-\mu} \tilde{u}_{-}^{\nu} \nabla_{\nu} \tilde{u}_{-}^{\mu}\right) .
$$

In the BTZ case, the unit normals are given by

$$
\begin{aligned}
\tilde{u}_{ \pm}^{\mu} & =\left(\sqrt{g_{E \pm}^{t t}}, 0, \frac{g_{E \pm}^{t \phi}}{\sqrt{g_{E \pm}^{t t}}}\right), \\
\tilde{n}_{ \pm \mu} & =\left(\mp \dot{R}, \pm \dot{t}_{E}, 0\right) .
\end{aligned}
$$

Then, on $\mathcal{W}, \tilde{u}_{ \pm}^{\phi}=0$, and we obtain

$$
\begin{aligned}
\left.n_{ \pm \mu} \tilde{u}_{ \pm}^{\nu} \nabla_{\nu} \tilde{u}_{ \pm}^{\mu}\right|_{\mathcal{W}} & =\left.\left(-\dot{R} \tilde{u}^{t_{E}} \nabla_{t_{E}} \tilde{u}^{t_{E}}+\dot{t}_{E} \tilde{u}^{t_{E}} \nabla_{t_{E}} \tilde{u}^{r}\right)\right|_{\mathcal{W}} \\
& =-\left.\frac{\partial_{r} f_{E \pm}}{2} \dot{t}_{E \pm}\right|_{\mathcal{W}},
\end{aligned}
$$

where we used

$$
\Gamma_{t_{E} t_{E}}^{r}=-\frac{f_{E}}{2} \partial_{r} f_{E}
$$

By using the trace of the 2nd junction condition 2.16

$$
[K]_{ \pm}=-16 \pi \sigma
$$

we get

$$
S_{\mathcal{M}_{+}}+S_{\mathcal{M}_{-}}=-2 \int_{\mathcal{W}} d^{2} x R \sigma-\frac{1}{16 \pi} \int_{\mathcal{W}} d^{2} x R\left(\partial_{r} f_{E+} \dot{t}_{E+}-\partial_{r} f_{E-} \dot{t}_{E-}\right) .
$$

Summing up all contributions, we rewrite the Euclidean action as

$$
\begin{aligned}
S_{E} & =-\frac{A_{\mathcal{H}_{-}}}{4}-2 \int_{\mathcal{W}} d^{2} x R \sigma-\frac{1}{16 \pi} \int_{\mathcal{W}} d^{2} x R\left(\partial_{r} f_{E+} \dot{t}_{E+}-\partial_{r} f_{E-} \dot{t}_{E-}\right)+\int_{\mathcal{W}} d^{2} x R \sigma \\
& =-\frac{A_{\mathcal{H}}}{4}-\frac{1}{16 \pi} \int_{\mathcal{W}} d^{2} x\left[\left(R \partial_{r} f_{E+}-2 f_{E+}\right) \dot{t}_{E+}-\left(R \partial_{r} f_{E-}-2 f_{E-}\right) \dot{t}_{E-}\right] \\
& =-\frac{A_{\mathcal{H}_{-}}}{4}-\frac{1}{8} \oint d \tau\left[\left(R \partial_{r} f_{E+}-2 f_{E+}\right) \dot{t}_{E+}-\left(R \partial_{r} f_{E-}-2 f_{E-}\right) \dot{t}_{E-}\right] \\
& =-\frac{A_{\mathcal{H}-}}{4}-2 \oint d \tau\left[\left(M_{+}+\frac{4 J_{E+}^{2}}{R^{2}}\right) \dot{t}_{E+}-\left(M_{-}+\frac{4 J_{E-}^{2}}{R^{2}}\right) \dot{t}_{E-}\right],
\end{aligned}
$$


where we used the relation

$$
R \sigma=-\frac{1}{8 \pi}\left[f_{E} \dot{t}_{E}\right]_{ \pm}
$$

given by the $(\phi, \phi)$ component of Eq. 2.22). By using $S_{E 0}=-\frac{A_{\mathcal{H}_{+}}}{4}$, we obtain

$$
\mathcal{B}=\frac{A_{\mathcal{H}_{+}}}{4}-\frac{A_{\mathcal{H}_{-}}}{4}-2 \oint d \tau\left[\left(M_{+}-\frac{4 J^{2}}{R^{2}}\right) \dot{t}_{E+}-\left(M_{-}-\frac{4 J^{2}}{R^{2}}\right) \dot{t}_{E-}\right] .
$$

The horizon area is

$$
\begin{aligned}
A_{\mathcal{H}_{ \pm}} & =\int_{0}^{2 \pi} d \phi r_{\mathcal{H}_{ \pm}} \\
& =2 \pi r_{\mathcal{H}_{ \pm}} .
\end{aligned}
$$

Then we can rewrite $\mathcal{B}$ as

$$
\mathcal{B}=\frac{\pi}{2}\left(r_{\mathcal{H}_{+}}-r_{\mathcal{H}_{-}}\right)-2 \oint d \tau\left[\left(M_{+}-\frac{4 J^{2}}{R^{2}}\right) \dot{t}_{E+}-\left(M_{-}-\frac{4 J^{2}}{R^{2}}\right) \dot{t}_{E-}\right] .
$$

\section{Results}

In this section, we show the parameter dependence of the nucleation rate for the cases which satisfy the conditions listed in Sec. 3. First, we evaluate the nucleation rate for $\tilde{a}_{+}=0$ and clarify the case which gives the dominant contribution to the tunnelling. Next, we introduce the angular momentum and see how the nucleation rate of the dominant case changes depending on the value of $\tilde{a}_{+}$.

\section{$5.1 \quad \tilde{a}_{+}=0$ case}

First, we set $\tilde{a}_{+}=0$. Setting $M_{+}=0.1, L_{+}=2.0$ and $s=0.2$, we plot the potential form for each value of $M_{-}$in Fig. 6. The available region for the shell motion gets smaller for a smaller value of $M_{-}$.

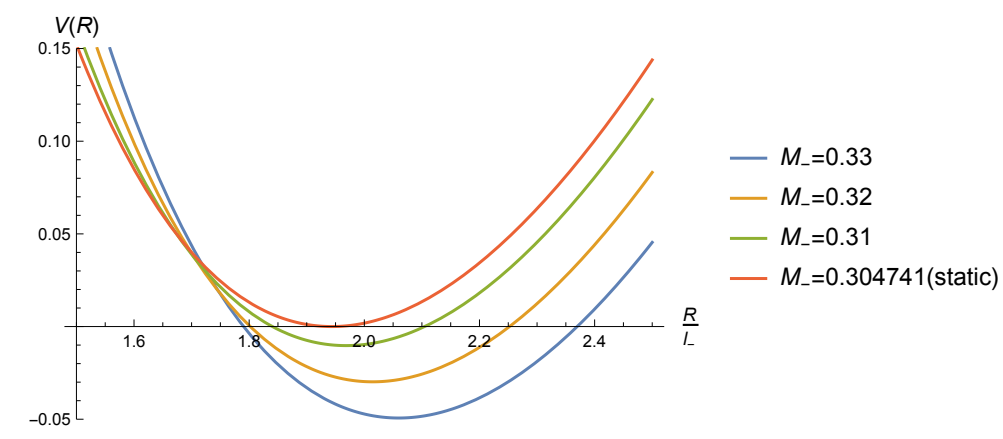

Figure 6: The potential form for $\tilde{a}_{+}=0, M_{+}=0.1, L_{+}=2.0, s=0.2$

Setting $\tilde{a}_{+}=0, L_{+}=2.0$ and $s=0.2$, we show the $M_{-}$dependence of $\mathcal{B} / \mathcal{B}_{C D L}$ for $M_{+}=0.1,0.2$ and 0.05 in Figs. $7 \mathrm{a}$, $7 \mathrm{~b}$ and $7 \mathrm{c}$, respectively (see App. A for the definition of $\mathcal{B}_{C D L}$ ). For all the cases, the left boundary of the plot region is set to the value of $M_{-}$for the static shell. We can see that, with a fixed value of $M_{+}$, the static shell case has the largest nucleation probability, and the rate is larger for a smaller value of $M_{+}$. This result does not change even if we change the value of the AdS length $L_{+}$as is explicitly shown for the case $M_{+}=0.1, L_{+}=1.7$ and $s=0.2$ in Fig. $7 \mathrm{~d}$ 


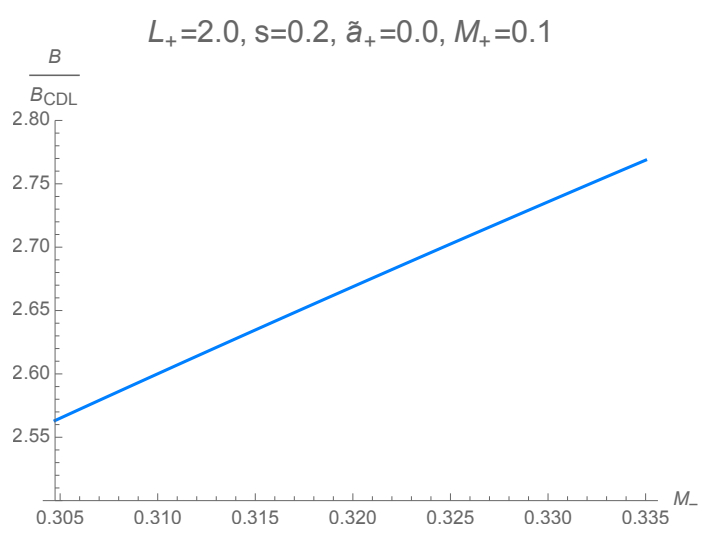

(a)

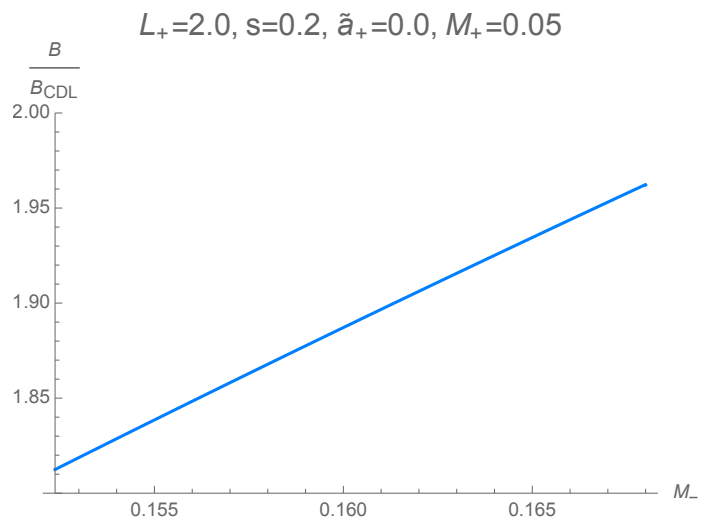

(c)

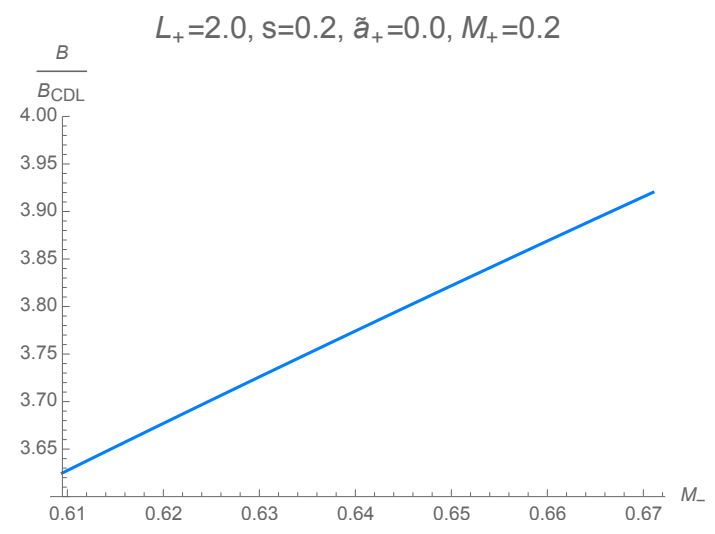

(b)

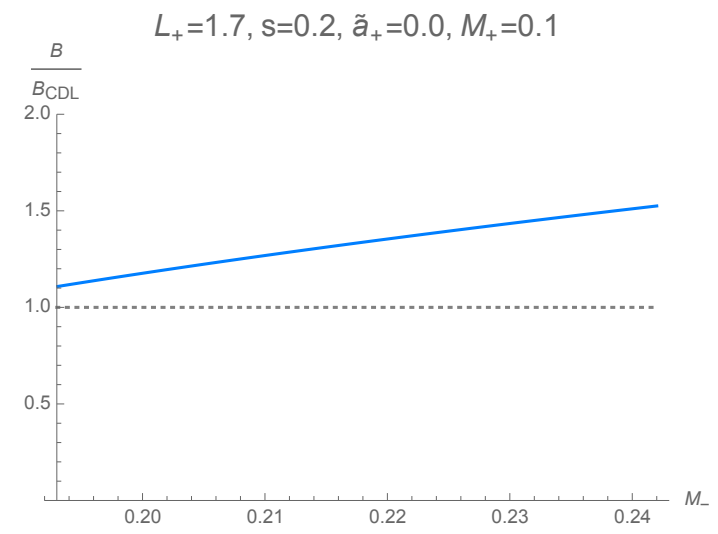

(d)

Figure 7: The $M_{-}$dependence of $\mathcal{B}\left(\tilde{a}_{+}=0\right)$

The $L_{+}=1.7$ case has a smaller value of $\mathcal{B} / \mathcal{B}_{C D L}$ than the $L_{+}=2.0$ case. We note that the value of $\mathcal{B} / \mathcal{B}_{C D L}$ is larger than unity in all cases shown in Figs. $7 \mathrm{a} / 7 \mathrm{~d}$.

The $M_{+}$dependence of $\mathcal{B}$ in the case of the static shell for each value of $L_{+}$is shown in Figs. $8 \mathrm{a}$ and $8 \mathrm{~b}$ with $s=0.2$ and $s=0.15$, respectively. Here, we note that the parameter sets of $\left(s, L_{+}\right)=(0.2,1.67)$ and $(0.15,1.43)$ almost saturate the inequality $(3.26)$. We can see that, with fixed $s$ and $L_{+}, \mathcal{B}$ in the static shell case increases monotonically with $M_{+}$. When the mass of the seed BH is small enough $\left(M_{+} \lesssim 0.02\right)$, we find $\mathcal{B}<\mathcal{B}_{C D L}$. This tendency is similar to the results in Refs. 5. 19. We can see that when we take the same values of $L_{+}$and $M_{+}$, a smaller value of $s$ gives a larger value of $\mathcal{B} / \mathcal{B}_{C D L}$. It should be noted that, in our case, differently from Refs. [5 19], the mass necessary increase through the nucleation, and the static shell case always exists. 


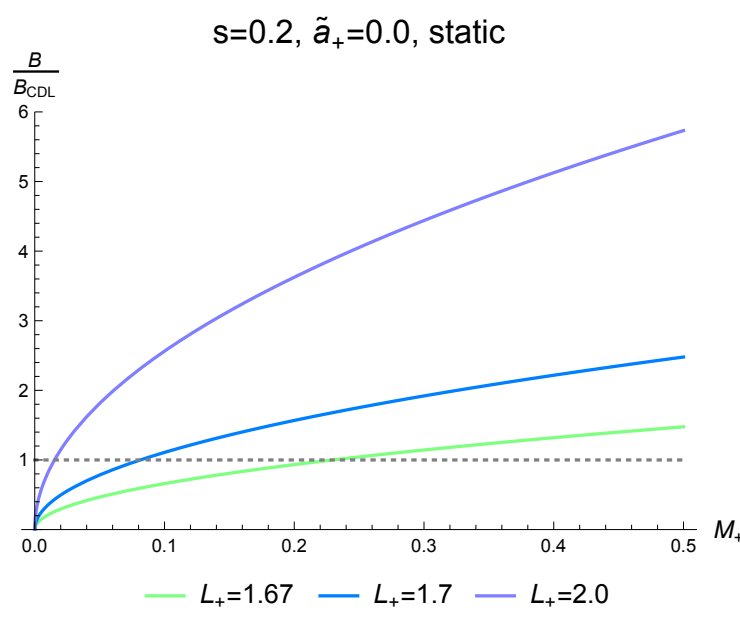

(a)

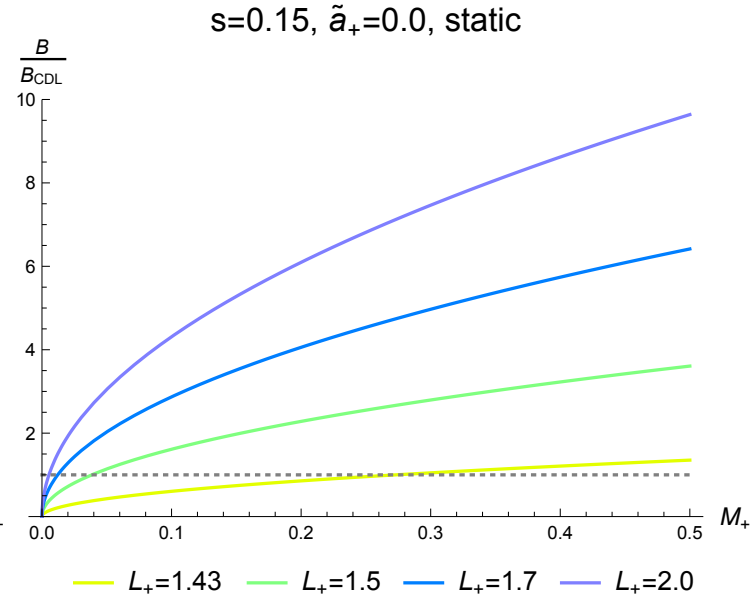

(b)

Figure 8: The $M_{+}$dependence of $\mathcal{B}$ of the static shells

\section{$5.2 \quad \tilde{a}_{+} \neq 0$ cases}

Next, we consider $\tilde{a}_{+} \neq 0$ cases. The potential form with $M_{+}=0.1, M_{-}=0.32, L_{+}=2.0$ and $s=0.2$ is shown for each value of $\tilde{a}_{+}$in Fig. 9. We can see that increasing $\tilde{a}_{+}$deepens the potential depth. The numerical result for $\mathcal{B}$ is shown in Fig. 10. The behavior is similar to the $\tilde{a}_{+}=0$ cases, and the formation of the static shell dominates the nucleation probability.

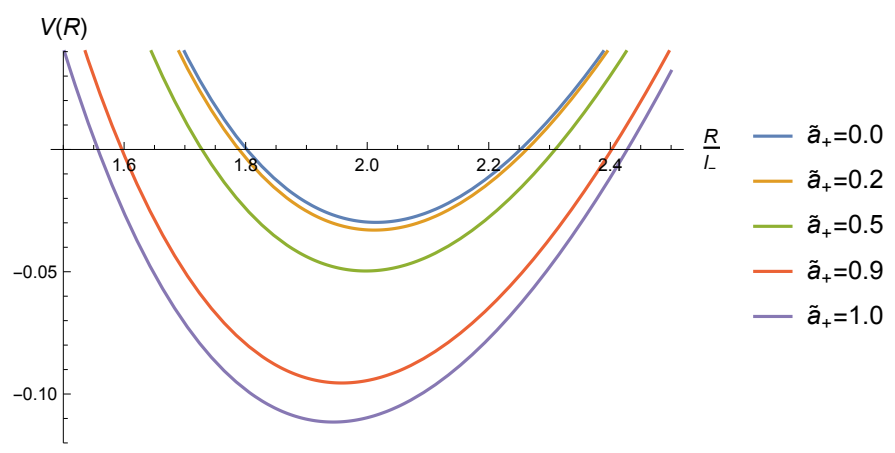

Figure 9: The potential form for $M_{+}=0.1, M_{-}=0.32, L_{+}=2.0$ and $s=0.2$ 

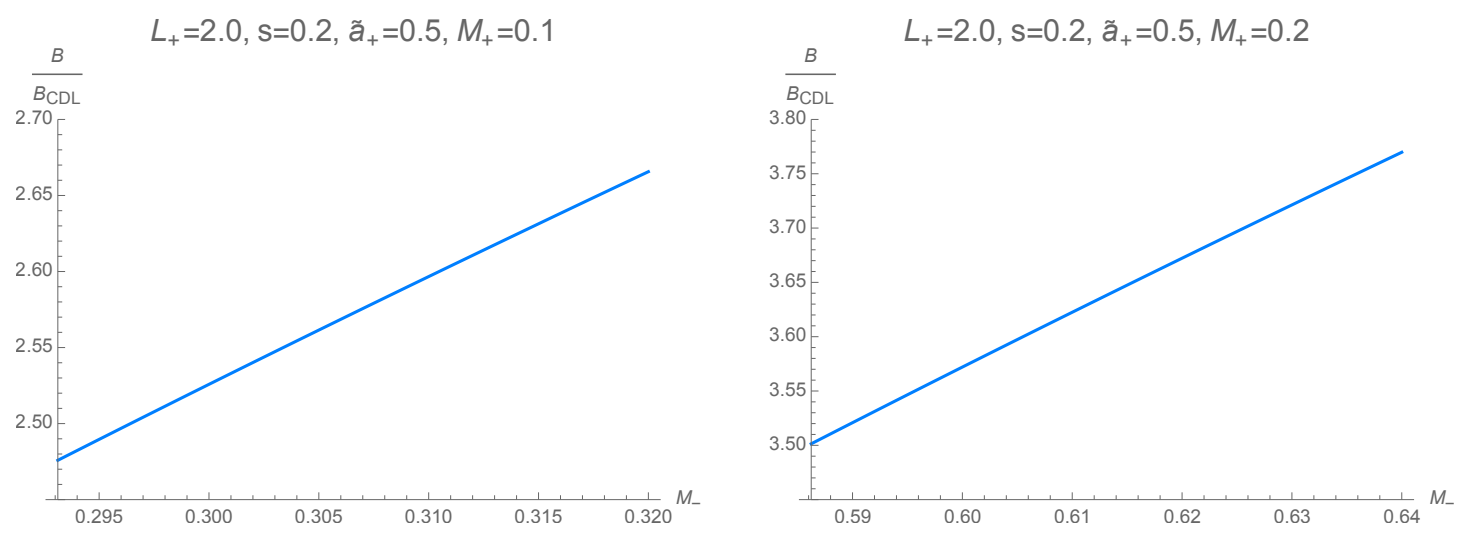

Figure 10: The $M_{-}$dependence of $\mathcal{B}\left(\tilde{a}_{+} \neq 0\right)$

Motivated by the results that static shells dominate even for $\tilde{a}_{+} \neq 0$, we consider the $\tilde{a}_{+}$dependence of the decay rate of the static shell. Let us define $M_{s}$ as the value of $M_{-}$for the static shell case. Then $M_{s}$ decreases with $\tilde{a}_{+}$(Fig. 11), as expected from Fig. 9. The $\tilde{a}_{+}$dependence of $\mathcal{B}$ for the static shell case is shown in Fig. 12 and we see that increasing $\tilde{a}_{+}$raises the decay rate.

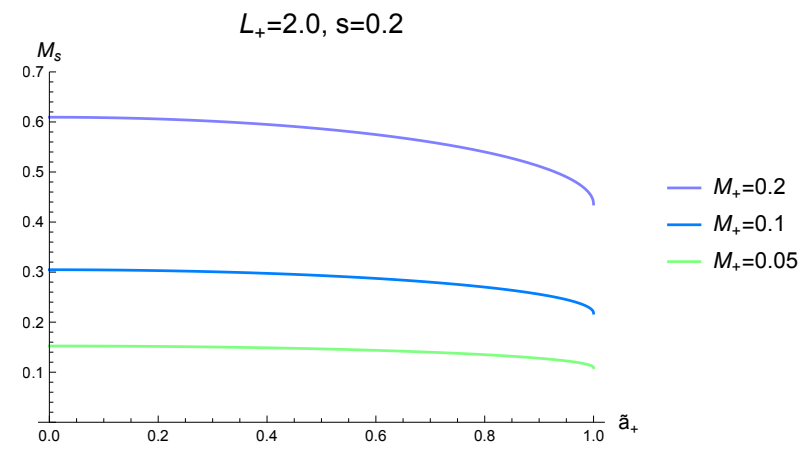

Figure 11: The $\tilde{a}_{+}$dependence of $M_{-}$of static shells
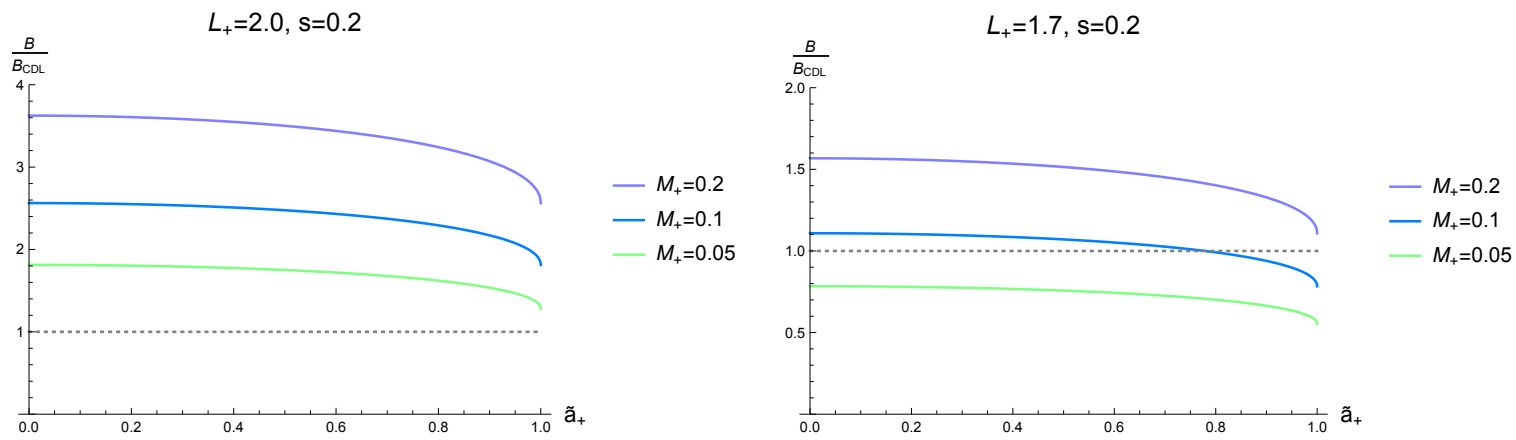

Figure 12: The $\tilde{a}_{+}$dependence of $\mathcal{B}$ of static shells 


\section{Summary and Discussion}

In this paper, we have analysed vacuum decay in rotating BTZ BH spacetimes. In the computation, we have used the thin shell approximation and the Israel's junction condition to see the motion of the bubble wall.

As a result, we found that the decay rate can be lower than that of the Coleman De Luccia Instanton. We found that the decay rate is a decreasing function of $M_{-}$and takes the maximum value at the lowest possible value of $M_{-}$, which is the case of the static shell. This result is partially consistent with results in Refs. 5. 6]. In these previous works, however, in relatively small $M_{+}$cases, bubble nucleation with no $\mathrm{BH}$ remnant dominates the probability. This difference stems from the difference in the change of the $\mathrm{BH}$ mass through the bubble nucleation. In the previous works, the BH mass can either increase or decrease with the nucleation, depending on the value of $M_{+}$. In particular, the black hole mass decreases for the small $M_{+}$ region and there is no static shell configuration for a sufficiently small $M_{+}$in the cases treated in Refs. [5,6]. In our cases, on the other hand, the $\mathrm{BH}$ mass must increase through bubble nucleation, and we can always have a static shell configuration (see App. $\mathrm{D}$ for the non-existence of the transition to a spacetime without a $\mathrm{BH}$ remnant).

We also found that the decay rate is a monotonic increasing function of $\tilde{a}_{+}$. That is, the angular momentum of the BTZ BH promotes the vacuum decay. On the other hand, the previous work on the Kerr spacetime 24] stated that the angular momentum suppresses the vacuum decay rate. Our results cannot be directly compared with those in Ref. 24] because of the totally different background settings and the assumptions 3 . The analyses in the background Kerr spacetime are much harder than the analyses given here because of the lack of symmetry. Obviously, more investigations about the non-spherical bubble nucleation process would be needed to understand the effects of the angular momentum.

In this work, focusing on vacuum decay in the BTZ spacetime, we have found several non-trivial results which cannot be expected from the previous works. However, we do not have a clear understanding about the physical interpretation of these results, and more multidirectional study would be needed.

\section{Acknowledgements}

This work was supported by JSPS KAKENHI Grant Numbers JP19H01895 (C.Y.), JP20H05850 (C.Y.) and JP20H05853 (C.Y.).

\section{A Three-Dimensional Coleman De Luccia Instanton}

In this appendix, we discuss vacuum decay in the pure AdS spacetime. The line element in the threedimensional AdS spacetime is given by

$$
d s^{2}=-h(r) d t^{2}+\frac{1}{h(r)} d r^{2}+r^{2} d \phi^{2}
$$

where

$$
h(r)=1+\frac{r^{2}}{l^{2}} .
$$

As in the BTZ case, we set the shell $\mathcal{W}: r-R\left(t_{E}\right)=0$ and consider the junction conditions. From the $(\phi, \phi)$ component of the 2 nd condition, we get

$$
-\frac{1}{2} \dot{R}^{2}:=U(R)=\frac{1}{2} \bar{\sigma}^{2} R^{2}-\frac{1}{2} \bar{h}+\frac{(\Delta h)^{2}}{32 \bar{\sigma}^{2} R^{2}},
$$

\footnotetext{
${ }^{3}$ The authors in Ref. 24 focus on the cases $M_{+}=M_{-}, a_{+}=a_{-}, a_{ \pm}^{2} / l^{2} \ll 1$, where the 1st junction conditions are automatically satisfied without angular dependencee. Then they introduce anisotropic tension to the stress energy tensor of the shell so that the 2nd junction condition can be satisfied. The form of the energy momentum tensor is clearly different from the isotropic tension adopted in our setting. We also note that the use of the Kerr-AdS geometry could be a nontrivial assumption because of the lack of the Birkhoff's theorem without spherical symmetry.
} 
and by using the form of $h(r)$, we rewrite $U(R)$ as

$$
U(R)=\frac{1}{2} R^{2}\left[\left\{\bar{\sigma}-\frac{1}{4 \bar{\sigma}}\left(\frac{1}{l_{+}^{2}}-\frac{1}{l_{-}^{2}}\right)\right\}^{2}-\frac{1}{l_{-}^{2}}\right]-\frac{1}{2} .
$$

This is the potential that governs the motion of the Euclidean shell. The functional form of $U(R)$ is depicted in Fig. 13 for $L_{+}=2.0$, and $s=0.2$.

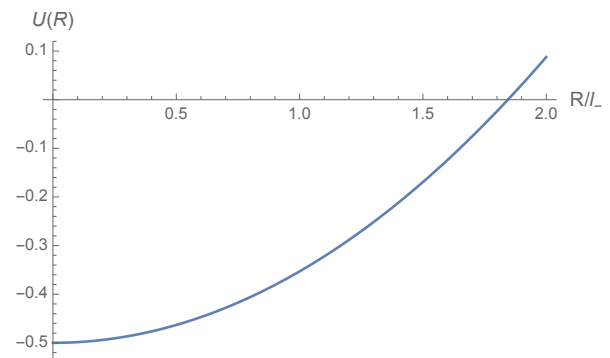

Figure 13: Example of the potential of CDL instanton $\left(L_{+}=2.0, s=0.2\right)$

We estimate the decay rate using the Euclidean action. Similarly to the BTZ case, we obtain the unit normal vectors $\tilde{u}_{ \pm}^{\mu}$ and $\tilde{n}_{ \pm \mu}$ as follows:

$$
\begin{aligned}
\tilde{u}_{ \pm}^{\mu} & =\left(\sqrt{g_{E \pm}^{t t}}, 0,0\right), \\
\tilde{n}_{ \pm \mu} & =\left(\mp \dot{R}, \pm \dot{t}_{E}, 0\right) .
\end{aligned}
$$

Then we get

$$
\begin{aligned}
\left.\tilde{n}_{ \pm \mu} \tilde{u}_{ \pm}^{\nu} \nabla_{\nu} \tilde{u}_{ \pm}^{\mu}\right|_{\mathcal{W}} & =\left.\left(\mp \dot{R} \tilde{u}^{t_{E}} \nabla_{t_{E}} \tilde{u}^{t_{E}} \pm \dot{t}_{E} \tilde{u}^{t_{E}} \nabla_{t_{E}} \tilde{u}^{r}\right)\right|_{\mathcal{W}} \\
& =\left.\mp \dot{t}_{E \pm}\left(-\frac{R}{l_{ \pm}^{2}}\right)\right|_{\mathcal{W}},
\end{aligned}
$$

where we used

$$
\Gamma_{t_{E} t_{E}}^{r}=-h(r) \frac{R}{l^{2}}
$$

Then we get the bulk action as follows,

$$
S_{\mathcal{M}_{+}}+S_{\mathcal{M}_{-}}=-2 \int_{\mathcal{W}} d^{2} x R \sigma-\frac{1}{8 \pi} \int_{\mathcal{W}} d^{2} x R\left(\frac{R}{l_{+}^{2}} \dot{t}_{E+}-\frac{R}{l_{-}^{2}} \dot{t}_{E-}\right) .
$$

Because there is no horizon, $S_{\mathcal{H}}=0$, and we have the same $S_{\mathcal{W}}$ as the BTZ case. Finally, we obtain the following expression of the exponential factor:

$$
\begin{aligned}
\mathcal{B}_{C D L} & =S_{E} \\
& =-\int_{\mathcal{W}} d^{2} x R \sigma-\frac{1}{8 \pi} \int_{\mathcal{W}} d^{2} x R\left(\frac{R}{l_{+}^{2}} \dot{t}_{E+}-\frac{R}{l_{-}^{2}} \dot{t}_{E-}\right) \\
& =\frac{1}{4} \oint d \tau\left(\dot{t}_{E+}-\dot{t}_{E-}\right) \\
& =\frac{1}{2}\left(L_{+} \sinh ^{-1} \frac{4 L_{+} s}{\sqrt{1+L_{+}^{4}\left(1-4 s^{2}\right)^{2}-2 L_{+}^{2}\left(1+4 s^{2}\right)}}-\sinh ^{-1} \frac{4 L_{+}^{2} s}{\sqrt{1+L_{+}^{4}\left(1-4 s^{2}\right)^{2}-2 L_{+}^{2}\left(1+4 L_{+} s^{2}\right)}}\right)
\end{aligned}
$$


where we used

$$
R \sigma=-\frac{1}{8 \pi}\left[h \dot{t}_{E}\right]_{ \pm}
$$

that is derived from the $(\phi, \phi)$ component of Eq. 2.22.

\section{B Static Shell}

Here, we consider the static shell as a special case of the bounces. We can get this special solution by requiring $\dot{R}=0=\partial_{t_{E}} R$. In this case, we have

$$
n_{\mu}=\left(0, \frac{1}{\sqrt{f_{E}(r)}}, 0\right) .
$$

From the normalization, we have $\frac{1}{\sqrt{f_{E}(r)}}=\dot{t}_{E}$, and

$$
\begin{aligned}
n_{\mu} & =\left(0, \dot{t}_{E}, 0\right), \\
n^{\mu} & =\left(0, \frac{1}{\dot{t}_{E}}, 0\right) .
\end{aligned}
$$

Using this unit normal vector, the $(\phi, \phi)$ component of the extrinsic curvature is calculated as

$$
K_{\phi \phi}=\sqrt{f_{E}(r)}
$$

and from Eq. (2.22), we have the following equation:

$$
\left.\left[\sqrt{f_{E+}(r)}-\sqrt{f_{E-}(r)}\right]\right|_{r=R}=-2 \bar{\sigma} R .
$$

This equation is equivalent to $V(R)=0$. The $(\tau, \tau)$ component of Eq. 2.22 is

$$
\left.\left[\frac{\partial_{r} f_{E+}(r)}{2 \sqrt{f_{E+}(r)}}-\frac{\partial_{r} f_{E-}(r)}{2 \sqrt{f_{E-}(r)}}\right]\right|_{r=R}=-2 \bar{\sigma},
$$

which is equivalent to $R$ derivative of Eq. B.5, $V^{\prime}(R)=0$.

Next, we compute $\mathcal{B}$ for the static case. The unit normal vectors are written as

$$
\begin{aligned}
\tilde{u}_{ \pm}^{\mu} & =\left(\sqrt{g_{E \pm}^{t t}}, 0, \frac{g_{E \pm}^{t \phi}}{\sqrt{g_{E \pm}^{t t}}}\right), \\
\tilde{n}_{ \pm \mu} & =\left(0, \pm \dot{t}_{E}, 0\right),
\end{aligned}
$$

so that $\tilde{u}_{ \pm}^{\mu} \tilde{n}_{ \pm \mu}=0$. Hence, we get

$$
\begin{aligned}
\frac{1}{8 \pi} \int_{\mathcal{W}} d^{2} x \sqrt{h_{E}}\left(n_{+\mu} \tilde{u}_{+}^{\nu} \nabla_{\nu} \tilde{u}_{+}^{\mu}-n_{-\mu} \tilde{u}_{-}^{\nu} \nabla_{\nu} \tilde{u}_{-}^{\mu}\right) & =-\frac{1}{8 \pi} \int_{\mathcal{W}} d^{2} x \sqrt{h_{E}}\left(\tilde{u}_{+}^{\mu} \tilde{u}_{+}^{\nu} \nabla_{\nu} n_{+\mu}-\tilde{u}_{-}^{\mu} \tilde{u}_{-}^{\nu} \nabla_{\nu} n_{-\mu}\right) \\
& =-\frac{1}{8 \pi} \int_{\mathcal{W}} d^{2} x \sqrt{h_{E}}\left(K_{+\tau \tau}-K_{-\tau \tau}\right) .
\end{aligned}
$$

Here, from Eq. 2.22,

$$
K_{E+\tau \tau}-K_{E-\tau \tau}=-8 \pi \sigma
$$


is satisfied, and we can write the bulk action as

$$
\begin{aligned}
S_{\mathcal{M}_{+}}+S_{\mathcal{M}_{-}} & =\frac{1}{8 \pi} \int_{\mathcal{W}} d^{2} x \sqrt{h_{E}}(-16 \pi \sigma)-\frac{1}{8 \pi} \int_{\mathcal{W}} d^{2} x \sqrt{h_{E}}(-8 \pi \sigma) \\
& =-\int_{\mathcal{W}} d^{2} x \sqrt{h_{E}} \sigma .
\end{aligned}
$$

This is the same as $S_{\mathcal{W}}$ in Eq. 4.4 with the opposite sign, and they cancel out. Eventually, the only nonzero contribution to $S_{E}$ is from $S_{\mathcal{H}_{-}}$.

The contribution from the false vacuum can be written as

$$
S_{E 0}=-\frac{A_{\mathcal{H}_{+}}}{4}
$$

so that we can write $\mathcal{B}$ as

$$
\mathcal{B}=\frac{1}{4}\left(A_{\mathcal{H}_{+}}-A_{\mathcal{H}_{-}}\right)
$$

which indicates that the decay rate depends only on the $\mathrm{BH}$ horizon areas. We note that this simple result comes from the relation $\tilde{u}_{ \pm}^{\mu} \tilde{n}_{ \pm \mu}=0$, originated from the staticness of the shell.

\section{Horizon Area}

In this appendix, we show that the horizon area decreases through the nucleation under the conditions in Sec. 3. Since the spatial section of the horizon is a one-dimensional object, its change is proportional to the difference of the radius, that is,

$$
\begin{aligned}
\frac{1}{l_{-}} \Delta r_{\mathcal{H}} & :=\frac{1}{l_{-}}\left(r_{\mathcal{H}_{+}}-r_{\mathcal{H}_{-}}\right) \\
& =\sqrt{4 M_{+} L_{+}^{2}+4 M_{+} L_{+}^{2} \sqrt{1-\tilde{a}_{+}^{2}}}-\sqrt{4 M_{-}+4 \sqrt{M_{-}^{2}-M_{+}^{2} L_{+}^{2} \tilde{a}_{+}^{2}}} .
\end{aligned}
$$

From Eq. [C.1], for $\Delta r_{\mathcal{H}}$ to be positive, $M_{-}$must satisfy the condition

$$
M_{-}<M_{r}:=\frac{M_{+}}{2}\left[L_{+}^{2}+1+\left(L_{+}^{2}-1\right) \sqrt{1-\tilde{a}_{+}^{2}}\right] .
$$

By comparing $M_{r}$ with $M_{T}$, which gives the upper bound of $M_{-}$from the condition $\dot{t}_{E \pm}>0$, we find

$$
M_{r}-M_{T}=2 M_{+} L_{+}^{2} s^{2}\left[1+\sqrt{1-\tilde{a}_{+}^{2}}\right]>0 .
$$

Therefore, as long as we focus on the nucleation with $M_{-}<M_{T}$, the horizon area decrease. Meanwhile, for a shrinking solution after the nucleation, the horizon area increases through the classical process of the shell accretion.

\section{Possibility of Other Bounce Solutions}

Let us consider the possibility of other bounce solutions. First, we consider the case $A<0, C>0$ and $\dot{t}_{E \pm}>0$. The potential form is given like Fig. 14. In this case, although we may consider Euclidean shell motion in the region $V(R)<0$, after the nucleation, the Lorentzian shell shrinks. Thus we do not consider this case. 


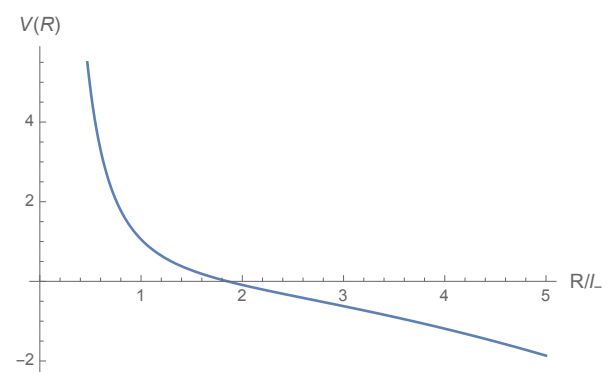

Figure 14: The potential form for $L_{+}=2.0, s=0.3, \tilde{a}_{+}=0, M_{+}=0.1$ and $M_{-}=0.336$

Next, we consider the case $A>0, C<0$ and $\dot{t}_{E \pm}>0$. In this case, the potential form is given like Fig. 15. About the motion of the Euclidean bounce, one might expect that a shell emerges from the one point $R=0$, and expands until the turn around point at $V(R)=0$, similarly to the CDL case. In the BH case, however, the point $R=0$ corresponds to the singularity, and the bounce solution is also singular at $R=0$. Therefore we do not consider this case.

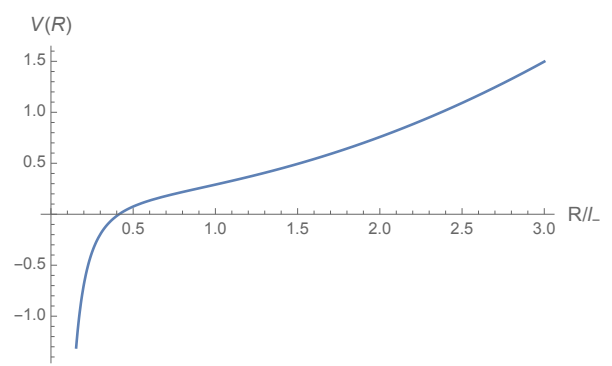

Figure 15: The potential form for $L_{+}=2.0, s=0.2, \tilde{a}_{+}=0, M_{+}=0.1$ and $M_{-}=0.13$ )

Finally, let us consider the transition between a BTZ and the pure AdS spacetime, which is given by $M=-\frac{1}{8}$ and $J=0$. There is two possibilities for this sort of nucleation : the transition between the BTZ spacetime and the pure AdS spacetime which corresponds to true/false vacuum.

First, we consider the former case, namely, $M_{-}=-\frac{1}{8}$ and $M_{+}>0$. By using Eq. (3.26), we obtain the lower bound for $B$ :

$$
\begin{aligned}
B & >4\left(M_{+}+M_{-}\right)-\frac{L_{+}^{-2}-1}{\frac{1}{4} L_{+}^{-2}-\frac{1}{2} L_{+}^{-1}+\frac{1}{4}}\left(M_{+}-M_{-}\right) \\
& =\frac{8\left(M_{+} L_{+}-M_{-}\right)}{L_{+}-1} .
\end{aligned}
$$

With $M_{-}=-\frac{1}{8}$, we see

$$
B>\frac{8 M_{+} L_{+}+1}{L_{+}-1}>0
$$

and $B$ cannot be negative and we cannot make a bounce solution. Next, we consider the the case $M_{+}=-\frac{1}{8}$ and $M_{-}>0$. By comparing $M_{D}$ and $M_{T}$, we obtain

$$
M_{D}-M_{T}=\frac{M_{+}}{2} \sqrt{1-\tilde{a}_{+}^{2}}\left[1+L_{+}^{2}\left(-1+4 s^{2}\right)+\sqrt{1+L_{+}^{4}\left(1-4 s^{2}\right)^{2}-2 L_{+}^{2}\left(1+4 s^{2}\right)}\right],
$$


and we see $1+L_{+}^{2}\left(-1+4 s^{2}\right)<0$ from Eq. (3.26). By noting that

$$
\begin{aligned}
& {\left[1+L_{+}^{2}\left(-1+4 s^{2}\right)\right]^{2}-\left[1+L_{+}^{4}\left(1-4 s^{2}\right)^{2}-2 L_{+}^{2}\left(1+4 s^{2}\right)\right]=16 L_{+}^{2} s^{2}>0,} \\
& 1+L_{+}^{2}\left(-1+4 s^{2}\right)-\sqrt{1+L_{+}^{4}\left(1-4 s^{2}\right)^{2}-2 L_{+}^{2}\left(1+4 s^{2}\right)}<0,
\end{aligned}
$$

we obtain $1+L_{+}^{2}\left(-1+4 s^{2}\right)+\sqrt{1+L_{+}^{4}\left(1-4 s^{2}\right)^{2}-2 L_{+}^{2}\left(1+4 s^{2}\right)}<0$. From that, we see $M_{T}<M_{D}$ when $M_{+}<0$, so there is no parameter region of $M_{-}$such that $M_{D}<M_{-}<M_{T}$. Instead of $M_{D}$, let us adopt $M_{D}^{\prime}$ as the bound for $M_{-}$. In this case,

$$
M_{C}-M_{D}^{\prime}=\frac{M_{+}}{2}\left[1+4 \tilde{a}_{+} L_{+} s+L_{+}^{2}\left(-1+4 s^{2}\right)+\sqrt{\left(1-\tilde{a}_{+}^{2}\right)\left\{1+L_{+}^{4}\left(1-4 s^{2}\right)^{2}-2 L_{+}^{2}\left(1+4 s^{2}\right)\right\}}\right]
$$

and $1+4 \tilde{a}_{+} L_{+} s+L_{+}^{2}\left(-1+4 s^{2}\right)<0$ follows from Eq. (3.26). By noting that

$$
\begin{aligned}
& {\left[1+4 \tilde{a}_{+} L_{+} s+L_{+}^{2}\left(-1+4 s^{2}\right)\right]^{2}-\left[\left(1-\tilde{a}_{+}^{2}\right)\left\{1+L_{+}^{4}\left(1-4 s^{2}\right)^{2}-2 L_{+}^{2}\left(1+4 s^{2}\right)\right\}\right]} \\
& =\left[\tilde{a}_{+}+4 L_{+} s+\tilde{a}_{+} L_{+}^{2}\left(-1+4 s^{2}\right)\right]^{2}>0, \\
& 1+4 \tilde{a}_{+} L_{+} s+L_{+}^{2}\left(-1+4 s^{2}\right)-\sqrt{\left(1-\tilde{a}_{+}^{2}\right)\left\{1+L_{+}^{4}\left(1-4 s^{2}\right)^{2}-2 L_{+}^{2}\left(1+4 s^{2}\right)\right\}}<0,
\end{aligned}
$$

we obtain $M_{D}^{\prime}<M_{C}$ for $M_{+}<0$. Then there is no parameter region of $M_{-}$such that $M_{C}<M_{-}<M_{D}^{\prime}$. Therefore there is not the case in which the potential form is given like Fig. 1.

From the above results, we conclude that the cases we considered in the main part of this paper are the only possible physical bounces in BTZ spacetimes.

\section{References}

[1] Sidney R. Coleman. The Fate of the False Vacuum. 1. Semiclassical Theory. Phys. Rev. D, 15:2929-2936, 1977. [Erratum: Phys.Rev.D 16, 1248 (1977)].

[2] Curtis G. Callan, Jr. and Sidney R. Coleman. The Fate of the False Vacuum. 2. First Quantum Corrections. Phys. Rev. D, 16:1762-1768, 1977.

[3] Sidney R. Coleman and Frank De Luccia. Gravitational Effects on and of Vacuum Decay. Phys. Rev. $D, 21: 3305,1980$.

[4] W. A. Hiscock. CAN BLACK HOLES NUCLEATE VACUUM PHASE TRANSITIONS? Phys. Rev. $D, 35: 1161-1170,1987$.

[5] Ruth Gregory, Ian G. Moss, and Benjamin Withers. Black holes as bubble nucleation sites. JHEP, 03:081, 2014.

[6] Philipp Burda, Ruth Gregory, and Ian Moss. Vacuum metastability with black holes. JHEP, 08:114, 2015.

[7] Kyohei Mukaida and Masaki Yamada. False Vacuum Decay Catalyzed by Black Holes. Phys. Rev. D, 96(10):103514, 2017.

[8] Georges Aad et al. Observation of a new particle in the search for the Standard Model Higgs boson with the ATLAS detector at the LHC. Phys. Lett. B, 716:1-29, 2012.

[9] Serguei Chatrchyan et al. Observation of a New Boson at a Mass of $125 \mathrm{GeV}$ with the CMS Experiment at the LHC. Phys. Lett. B, 716:30-61, 2012. 
[10] Marc Sher. Electroweak Higgs Potentials and Vacuum Stability. Phys. Rept., 179:273-418, 1989.

[11] Peter B. Arnold. Can the electroweak vacuum be unstable? Phys. Rev. D, 40:613-619, Jul 1989.

[12] J. R. Espinosa and M. Quiros. Improved metastability bounds on the standard model Higgs mass. Phys. Lett. B, 353:257-266, 1995.

[13] Gino Isidori, Giovanni Ridolfi, and Alessandro Strumia. On the metastability of the standard model vacuum. Nucl. Phys. B, 609:387-409, 2001.

[14] Joan Elias-Miro, Jose R. Espinosa, Gian F. Giudice, Gino Isidori, Antonio Riotto, and Alessandro Strumia. Higgs mass implications on the stability of the electroweak vacuum. Phys. Lett. B, 709:222$228,2012$.

[15] Giuseppe Degrassi, Stefano Di Vita, Joan Elias-Miro, Jose R. Espinosa, Gian F. Giudice, Gino Isidori, and Alessandro Strumia. Higgs mass and vacuum stability in the Standard Model at NNLO. JHEP, 08:098, 2012.

[16] Dario Buttazzo, Giuseppe Degrassi, Pier Paolo Giardino, Gian F. Giudice, Filippo Sala, Alberto Salvio, and Alessandro Strumia. Investigating the near-criticality of the Higgs boson. JHEP, 12:089, 2013.

[17] So Chigusa, Takeo Moroi, and Yutaro Shoji. State-of-the-Art Calculation of the Decay Rate of Electroweak Vacuum in the Standard Model. Phys. Rev. Lett., 119(21):211801, 2017.

[18] So Chigusa, Takeo Moroi, and Yutaro Shoji. Decay Rate of Electroweak Vacuum in the Standard Model and Beyond. Phys. Rev. D, 97(11):116012, 2018.

[19] Philipp Burda, Ruth Gregory, and Ian Moss. Gravity and the stability of the Higgs vacuum. Phys. Rev. Lett., 115:071303, 2015.

[20] Philipp Burda, Ruth Gregory, and Ian Moss. The fate of the Higgs vacuum. JHEP, 06:025, 2016.

[21] Ruth Gregory and Ian G. Moss. The Fate of the Higgs Vacuum. PoS, ICHEP2016:344, 2016.

[22] Kazunori Kohri and Hiroki Matsui. Electroweak Vacuum Collapse induced by Vacuum Fluctuations of the Higgs Field around Evaporating Black Holes. Phys. Rev. D, 98(12):123509, 2018.

[23] De-Chang Dai, Ruth Gregory, and Dejan Stojkovic. Connecting the Higgs Potential and Primordial Black Holes. Phys. Rev. D, 101(12):125012, 2020.

[24] Naritaka Oshita, Kazushige Ueda, and Masahide Yamaguchi. Vacuum decays around spinning black holes. JHEP, 01:015, 2020. [Erratum: JHEP 10, 122 (2020)].

[25] Máximo Bañados, Claudio Teitelboim, and Jorge Zanelli. Black hole in three-dimensional spacetime. Phys. Rev. Lett., 69:1849-1851, Sep 1992.

[26] Máximo Bañados, Marc Henneaux, Claudio Teitelboim, and Jorge Zanelli. Geometry of the $2+1$ black hole. Phys. Rev. D, 48:1506-1525, Aug 1993.

[27] José P. S. Lemos, Francisco J. Lopes, and Masato Minamitsuji. Rotating thin shells in $(2+1)$ dimensional asymptotically AdS spacetimes: Mechanical properties, machian effects, and energy conditions. Int. J. Mod. Phys. D, 24(09):1542022, 2015.

[28] W. Israel. Singular hypersurfaces and thin shells in general relativity. Nuovo Cim. B, 44S10:1, 1966. [Erratum: Nuovo Cim.B 48, 463 (1967)].

[29] Constantin Bachas and Vassilis Papadopoulos. Phases of Holographic Interfaces. JHEP, 04:262, 2021.

[30] Constantin Bachas, Zhongwu Chen, and Vassilis Papadopoulos. Steady states of holographic interfaces. JHEP, 11:095, 2021. 